\section{P115 Food Security Among Households With Children Improved Following a Nutrition Education Intervention}

Rebecca L.Rivera, MPH, rcusack@purdue.edu, Purdue University, 700 West State Street, Stone Hall, West Lafayette, IN 47907; M. Maulding, MS, RD; A. Abbott, MA; H. Eicher-Miller, PhD

Objective: The effects of a Supplemental Nutrition Assistance Program-Education (SNAP-Ed) intervention on the food security of households with children in Indiana were determined.

Design, Setting and Participants: A randomized, controlled, intervention study design using SNAP-Ed as the intervention was implemented throughout 92 Indiana counties from $8 / 2013$ to $2 / 2014$. Five hundred sixty SNAPEd eligible participants $\geq 18 y$ with $\geq 1$ child residing in their household were recruited and randomly allocated to receive $\geq 4$ SNAP-Ed lessons or not during the 4 to 10 week study period, before and after which they also completed the 18-item US Household Food Security Survey Module to assess food security status and a characteristics questionnaire.

Outcome Measures and Analysis: Analysis of covariance modeling, adjusted for participant and household characteristics, was used to determine changes in household food security status ( $\mathrm{p} \leq 0.05$ ).

Results: The mean household food security improvement from before compared with after the study period was greater in the intervention $(-1.0, \mathrm{SD}=3.3)$ compared with the control group $(-0.4, \mathrm{SD}=2.7)$ in simple $(\mathrm{p}=0.04)$ and adjusted models $(\beta=-0.7, \mathrm{p}=0.03)$.

Conclusions and Implications: The SNAP-Ed program is an effective intervention to improve food security in households with children in Indiana over a short-term period. The results help build the evidence base to inform stakeholders about the impact of SNAP-Ed.

Funding: USDA, University of Kentucky Center for Poverty Research.

\section{P116 Determining the Need for a Food Pantry on a University Campus}

Karla Shelnutt, PhD, RD, kpagan@ufl.edu, University of Florida, 3038 McCarty Hall D, Gainesville, FL 32611

Objective: To determine food insecurity on a large university campus and to obtain input on preferences for a food pantry.

Design, Setting and Participants: An online survey with questions from the US Household Food Security Survey and questions developed by university faculty and staff to inform logistics for a food pantry was developed. All undergraduate and graduate students were invited to participate, and 1,891 students completed most or all of the survey in the fall 2014 semester.

Outcome Measures and Analysis: Descriptive statistics are provided.

Results: Ten percent of total respondents indicated they sometimes or often did not have enough food to eat, indicating food insecurity. Of these food insecure students,
$85 \%$ worried their food would run out before they got money to buy more, and 79\% indicated that the food they bought didn't last and they didn't have the money to get more. As a result of not having enough money for food, $81 \%$ cut the size of or skipped meals, $74 \%$ had been hungry but didn't eat, and 23\% did not eat for a whole day. In addition, $46 \%$ indicated that the inability to afford food affected their ability to learn, and $84 \%$ avoided dining out with friends because they could not afford the food. Eighty-seven percent of total respondents supported a food pantry and most preferred a hidden but central location on campus.

Conclusions and Implications: These data indicate a need and a desire for a food panty at a large university campus. University administrators, faculty and staff are now working together to start a sustainable, healthy food pantry.

Funding: None.

\section{P117 Intent to Use WIC Smartphone Application to Promote Breastfeeding, Physical Activity, and Healthy Eating in WIC Participants}

Lesli Biediger-Friedman, PhD, RD, MPH, lb47@txstate.edu, Texas State University, 601 University Drive, School of Family Consumer Science, San Marcos, TX 78666; M. Silva, BS; S. Crixell, PhD, RD; B. Friedman, PhD, RD, LDN; K. S. Smith, PhD

Objective: To examine performance expectancy of WIC app prototype usage toward improving health behaviors.

Design, Setting and Participants: A total of 54 WIC mothers in 6 focus groups held in Texas (79\% Hispanic, $42 \%$ Spanish preferred, 52\% rural, 22\% breastfeeding) tested a WIC app prototype. Themes based on performance expectancy constructs for technology acceptance were included in the focus group discussion guide. Observations were recorded and transcribed. Comments were identified and coded for themes based on performance expectancy constructs.

Outcome Measures and Analysis: Using Nvivo 10, examined constructs were perceived usefulness, outcome expectation, extrinsic motivation, and relative advantage. Results: Most participants said they would utilize a WIC app. Some mentioned the app would be useful to them specifically as mothers. Overall outcome expectation was that app use would increase healthy behaviors based on features such as planning, logging, and sharing information. Tracking and sharing progress were themes of extrinsic motivation. Perceived relative advantages were that a single app can promote multiple healthy behaviors and facilitate quick access to information.

Conclusions and Implications: Texas WIC mothers feel that a comprehensive app would facilitate physical activity, healthy eating and breastfeeding duration. Features that facilitated planning, enabled logging information, and provided quick access to resources were identified as especially useful. 
P117 (continued)

Funding: Texas Department of Health Services Contract with State WIC Program.

\section{P118 Home Food Environment Observations Using a Photo Voice Texting Method With Parents}

Lesli Biediger-Friedman, PhD, MPH, RD, 1b47@txstate.edu, Texas State University, 601 University Drive, School of Family and Consumer Sciences, San Marcos, TX 78666; K. Lines, BS; S. Crixell, PhD, RD; B. Friedman, PhD, RD

Objective: The nearly ubiquitous use of smartphones provides novel opportunities to assess home environments without significant time commitment or invasion of privacy. This study aimed to describe photo and text observations collected through self-assessments of home food behaviors and the home food environment.

Design, Setting and Participants: Forty-three parents (57\% non-Hispanic white; $35.7 \%$ Hispanic; $7.3 \%$ other) of a preschool aged child were recruited at childcare facilities in central Texas. For two weeks, researchers prompted participants via text to complete a series of self-assessments addressing food in the home and nutrition practices using grounded theory. Topics included: meal preparation, family mealtime, food in the home, goal-setting to improve family food and nutrition behaviors. Utilizing personal mobile phones, participants sent a photo and brief text reflection for each assignment. Participants were assisted in short and long-term goal setting to address identified nutrition barriers.

Outcome Measures and Analysis: Photos and reflections were analyzed thematically using NVivo 10.

Results: A common barrier included conflicting family schedules, which interfered with cooking and eating meals at home together. Food preferences for non-nutritional foods were a common barrier to providing fresh fruits and vegetables. Common goals included creating weekly menu plans, involving children in meal preparation and providing balanced meals based on family food preferences of fruits and vegetables.

Conclusions and Implications: Photo reflections prompted participants to increase their awareness of home eating behaviors and to promote positive changes with goal setting. Photo Voice activities may allow researchers and health professionals to connect with busy parents, potentially empowering parent's assessment of their home environments and recognize the need for change.

Funding: Texas State University, Research Enhancement Program.

\section{P119 Assessing the Feasibility of a Mobile Fresh Food Market}

JenniferWalsh,PhD, RD, jenniferwalsh@ufl.edu, University of Florida, Department of Family, Youth and Community Sciences, 1408 Sable Palm Drive, Gainesville, FL 32611; K. Shelnutt, PhD, RD; T. Johns, PhD;

M. Scicchitano, PhD; B. Waite, PhD
Objective: Individuals with limited resources living in food deserts lack access to affordable fresh fruits and vegetables and other foods needed for a healthful diet. The purpose of this research was to determine the feasibility of a mobile fresh food market (MFFM) that accepts Electronic Benefits Transfer for residents of food deserts in Gainesville, Florida.

Design, Setting and Participants: An in-person survey was conducted with a convenient sample of 1,200 residents, over 18 years old, of food deserts.

Outcome Measures and Analysis: The USDA Adult Food Security Survey and additional items related to eating behavior, willingness to purchase fresh produce from a MFFM, and demographics. Descriptive statistics were conducted.

Results: Over 70\% of respondents reported household incomes $<\$ 25,000$; almost half worried monthly whether food would run out before more money was available; $39 \%$ indicated their children were not eating enough because they could not afford enough food. Half reported eating fresh fruits and vegetables either every day or most days and almost $80 \%$ indicated they would shop for produce at a MFFM at least once a week.

Conclusions and Implications: Food insecurity exists in this population and a MFFM is desired to purchase fresh fruits and vegetables at a low cost. These data may be used to communicate the demand and supply needed for implementation of a MFFM.

Funding: Supplemental Nutrition Assistance Program Education.

\section{P120 Paint by Numbers of Incidence: What Depictions of Food in Paintings Can Tell Us About Aspirations and Aesthetics}

BrianWansink, PhD, foodandbrandlab@cornell.edu, Cornell University, 475 Warren Hall, Ithaca, NY 14853; A. Mukund, MS

Objective: Can the frequency a food is depicted in paintings give historical insight into family meals across countries and time? Paintings of large banquets tell us little about everyday food consumption in homes. It is unknown if these depictions represent historical accounts or the extent to which they vary across country and time. Design, Setting and Participants: While considering secular and religious paintings together, 140 paintings depicting family meals were content analyzed and indexed based on 91 food items from five countries (France, Germany, Italy, the Netherlands, and the United States) from $1500-2000$.

Outcome, Measures and Analysis: The religiosity and economic prosperity of a country were confounding factors. Incidence of each food item was coded and analyzed using $\chi 2$ analysis.

Results: Across countries and time $19.29 \%$ of the paintings included a vegetable, $75.71 \%$ contained a fruit, $38.57 \%$ contained a meat, and $41.43 \%$ contained bread. The variety of fruit was more heavily depicted than that of vegetables. Of the meats and indulgences analyzed, 